\title{
BRCA and BREAST CANCER RISK: A Bio-Environmental Susceptibility Study on Bengalee Females of India
}

\author{
Abhishikta Ghosh Roy $^{1}$ and A.R Bandyopadhyay ${ }^{2}$ \\ ${ }^{1}$ DNA Laboratory, Anthropological Survey of India, Kolkata \\ ${ }^{2}$ Human Genetics Laboratory, Department of Anthropology, University of Calcutta
}

\begin{abstract}
Breast cancer is considered to be the most frequent malignancy among women all over the world. The present study attempts to understand the molecular heterogeneity of BRCA1 and BRCA2 genes, as well as to understand the association of various lifestyle and reproductive variables for the Breast Cancer risk. The study was conducted in a total of 110 patients and 128 controls that revealed DNA sequencing of ten Single Nucleotide Polymorphisms (SNPs) $(6$ novel). Significant $(\mathrm{p}<0.005)$ molecular heterogeneity is revealed in terms of SNPs in BRCA1 (4 exonic \& 1 intronic variants) and BRCA2 (2exonic and 3 intronic variants) genes. The augmentation study revealed significant $(\mathrm{p}<0.005)$ association with positive family history, early age at menarche, irregular menstrual periods, menopause, prolong contraceptive use, nulliparity, history of abortions, consumption of alcohol and smoking towards breast cancer risk. This study being the first of its kind, envisaged that the identification of the SNPs and modification of the lifestyle factors might aid to minimize the risk among the Bengalee Hindu females.
\end{abstract}

Keywords: BRCA1, BRCA2, Breast cancer risk, Bengalee Hindu females, Lifestyle and Reproductive Variables, SNPs. 